\title{
Identification of genes involved in Epstein-Barr virus-associated nasopharyngeal carcinoma
}

\author{
JUNGUO WANG $^{1,2^{*}}$, FANG MEI $^{3 *}, \mathrm{XIA} \mathrm{GAO}^{1,2}$ and SHOULIN WANG ${ }^{4,5}$ \\ ${ }^{1}$ Department of Otolaryngology-Head and Neck Surgery, Drum Tower Clinical Medical College of Nanjing Medical \\ University, Nanjing, Jiangsu 210008; ${ }^{2}$ Department of Otolaryngology-Head and Neck Surgery, Nanjing Drum Tower \\ Hospital Affiliated to Nanjing University Medical School, Nanjing, Jiangsu 210008; ${ }^{3}$ Department of Ophthalmology, \\ Nanjing Children's Hospital Affiliated to Nanjing Medical University, Nanjing, Jiangsu 210008; ${ }^{4}$ Key Laboratory of \\ Modern Toxicology of Ministry of Education, School of Public Health, Nanjing Medical University; ${ }^{5}$ State Key Laboratory \\ of Reproductive Medicine, Institute of Toxicology, Nanjing Medical University, Nanjing, Jiangsu 211166, P.R. China
}

Received April 8, 2015; Accepted June 2, 2016

DOI: $10.3892 / \mathrm{ol} .2016 .4940$

\begin{abstract}
Nasopharyngeal carcinoma (NPC) is the most common cancer originating from the nasopharynx, and can be induced by infection with Epstein-Barr virus (EBV). To study the mechanisms of EBV-associated NPC, a microarray of the GSE12452 dataset was analyzed. GSE12452 was downloaded from Gene Expression Omnibus and consisted of 31 NPC samples and 10 normal healthy nasopharyngeal tissue samples. The differentially-expressed genes (DEGs) were screened using the linear models for microarray data package in R. Using Database for Annotation, Visualization and Integrated Discovery software, potential functions of the DEGs were predicted by Gene Ontology and pathway enrichment analyses. With the information from the Search Tool for the Retrieval of Interacting Genes/Proteins database, the protein-protein interaction (PPI) network was visualized by Cytoscape. Furthermore, modules of the PPI network were searched using ClusterONE in Cytoscape. A total of 951 DEGs were screened in the NPC samples compared with the normal healthy nasopharyngeal tissue samples. Function enrichment
\end{abstract}

Correspondence to: Dr Shoulin Wang, Key Laboratory of Modern Toxicology of Ministry of Education, School of Public Health, Nanjing Medical University, 101 Longmian Road, Jiangning, Nanjing, Jiangsu 211166, P.R. China

E-mail: shoulinwangslw@163.com

Dr Xia Gao, Department of Otolaryngology-Head and Neck Surgery, Drum Tower Clinical Medical College of Nanjing Medical University, 321 Zhongshan Road, Gulou, Nanjing, Jiangsu 210008, P.R. China

E-mail: xiagaoxgx@163.com

${ }^{*}$ Contributed equally

Key words: nasopharyngeal carcinoma, Epstein-Barr virus, differentially-expressed genes, protein-protein interaction network, module analysis indicated that the upregulated genes were associated with the cell cycle, cytoskeleton organization and DNA metabolism. Meanwhile, the downregulated genes were mainly associated with cell differentiation, hormone metabolism, inflammatory response and immune response. PPI networks for the DEGs suggested that upregulated mitotic arrest deficient 2-like 1 (MAD2L1; degree=133), proliferating cell nuclear antigen $(P C N A$; degree=125) and cyclin B1 (CCNB1; degree=115), and downregulated member $\mathrm{A} 1$ of aldehyde dehydrogenase 1 (ALDH1A1; degree=15) may be of great importance as they exhibited higher degrees on interaction. Mucin 1 (MUC1) was a key node of module 4 . Overall, the study indicated that MAD2L1, CCNB1, PCNA, ALDH1A1 and MUC1 may have a correlation with EBV-associated NPC.

\section{Introduction}

As the most common cancer originating from the nasopharynx (1), nasopharyngeal carcinoma (NPC) can be caused by viral influence, heredity and environmental factors (2). The viral influence is correlated with infection by Epstein-Barr virus (EBV), which is a B-lymphotropic herpes virus possessing growth-transforming properties (3). It has been reported that $95 \%$ of Americans are exposed to this virus in their thirties (4). In 2010, NPC led to 65,000 mortalities globally (5). Thus, there is an urgent requirement to study the mechanisms of EBV-associated NPC.

Recently, a number of studies have been performed to investigate the mechanisms of EBV-associated NPC. For example, as an early EBV antigen (6), BamH1-A Reading Frame-1 may be associated with the pathogenesis of NPC, such as the malignant transformation of human NPC epithelial cells $(7,8)$. Aberrant hypermethylation of Ras association domain family 1 isoform A and the high viral load of EBV DNA may play an important role in NPC pathogenesis, thus, they may function as promising diagnostic markers for NPC $(9,10)$. Serological results have shown that specifically expressed BRLF1 may be used in the diagnosis of NPC (11). Via the activation of Ets-1, c-Met can be induced by latent membrane protein-1 (LMP-1) and enhance the highly metastatic potential 
of NPC (12). By providing epitopes recognized by cytotoxic T-cells, EVB-encoded $L M P 2 A$ may be a potential target and may be used in the immunotherapy of NPC (13).

In 2006, Sengupta et al (14) analyzed the expression of all latent EBV genes between NPC samples and normal healthy nasopharyngeal epithelium samples, and obtained a panel of differentially-expressed genes (DEGs). Using the same data by Sengupta et al (14), the present study aimed to further screen the DEGs and predict their underlying function by functional and pathway enrichment analyses. Furthermore, protein-protein interaction network (PPI) networks were constructed and modules of PPI network were searched to investigate the interaction associations between these DEGs.

\section{Materials and methods}

Microarray data. The expression profile of the GSE12452 dataset deposited by Sengupta et al (14) was downloaded from Gene Expression Omnibus (http://www.ncbi.nlm.nih.gov/geo/), which was based on the platform of the GPL570 [HG-U133_Plus_2] Affymetrix Human Genome U133 Plus 2.0 Array. GSE12452 consisted of a collection of 31 NPC samples and 10 normal healthy nasopharyngeal tissue samples. These samples were collected from Taiwanese patients who provided informed consent. After the tissues were resected and immediately flash frozen, samples were finally stored in liquid nitrogen.

DEG screening. Once GSE12452 had been downloaded, the microarray data was read using Affy package (www.bioconductor.org) (15) and Refseq annotation files, and then it was normalized by the Robust MultiArray Averaging method (16). The linear models for microarray data package (http://www.bioconductor.org) (17) in R was used to identify the DEGs between NPC samples and normal healthy nasopharyngeal tissue samples. The adjusted P-value of $<0.01$ and |logfold-change (FC)|>1 were used as the cut-off criteria.

Functional and pathway enrichment analysis. Gene Ontology (GO) terms can describe three wild-type gene products, including molecular function, biological process and subcellular location (18). The Kyoto Encyclopedia of Genes and Genomes (KEGG) pathway database is used for the systematic analysis of gene functions, which can connect genomic information with functional information (19). The Database for Annotation, Visualization, and Integrated Discovery (DAVID) is a program that can integrate functional genomic annotations with intuitive summaries (20). Using DAVID software, GO and KEGG pathway enrichment analyses were performed for DEGs between NPC samples and normal healthy nasopharyngeal tissue samples. A P-value of $<0.05$ was used as the cut-off criterion.

PPI network and module construction. Interaction associations of the proteinsencoded by theDEGsweresearched by Search Tool for the Retrieval of Interacting Genes/Proteins online software (http://string-db.org/) (21), and then PPI networks were visualized by Cytoscape software (http://www.cytoscape.org) (22). The proteins in the PPI network were termed nodes and the degree of a node correlated with the number of its interactions.
Using network statistics, a connectivity degree analysis was conducted for each node in the PPI network. ClusterONE (http://www.paccanarolab.org/clusterone/) (23) in Cytoscape was used to screen modules of the PPI network. A P-value of $<0.01$ was used as the cut-off criterion.

\section{Results}

DEG analysis. Compared with normal healthy nasopharyngeal tissue samples, a total of 951 DEGs were screened from the NPC samples, including 376 upregulated and 575 downregulated genes. There were more downregulated genes than upregulated genes.

Functional and pathway enrichment analysis. The enriched GO functions for upregulated genes were divided into 28 clusters. Functions in cluster 1 were associated with the cell cycle, such as the $\mathrm{M}$ phase $\left(\mathrm{P}=5.27 \times 10^{-30}\right)$ and the cell cycle process $\left(\mathrm{P}=3.34 \times 10^{-29}\right)$. Functions in cluster 2 were associated with cytoskeleton organization, such as the microtubule-based process $\left(\mathrm{P}=1.09 \times 10^{-11}\right)$ and microtubule cytoskeleton organization $\left(\mathrm{P}=7.27 \times 10^{-11}\right)$. Functions in cluster 3 were implicated in the regulation of the cell cycle, such as the regulation of the mitotic cell cycle $\left(\mathrm{P}=6.43 \times 10^{-9}\right)$ and the regulation of cell cycle process $\left(\mathrm{P}=7.79 \times 10^{-6}\right)$. Functions in cluster 4 were involved in DNA metabolism, such as the DNA metabolic process $\left(\mathrm{P}=1.80 \times 10^{-12}\right)$ and the response to a DNA damage stimulus $\left(\mathrm{P}=5.83 \times 10^{-8}\right)$ (Table I).

The enriched GO functions for downregulated genes were divided into 9 clusters. For instance, functions in cluster 1 were associated with cell differentiation, such as epithelial cell differentiation $\left(\mathrm{P}=4.04 \times 10^{-7}\right)$ and keratinocyte differentiation $\left(\mathrm{P}=3.48 \times 10^{-3}\right)$. Functions in cluster 2 were involved in hormone metabolism, such as the regulation of hormone levels $\left(\mathrm{P}=5.75 \times 10^{-4}\right)$ and the cellular hormone metabolic process $\left(\mathrm{P}=4.25 \times 10^{-2}\right)$. Functions in cluster 3 were associated with the inflammatory response, such as the acute inflammatory response $\left(\mathrm{P}=1.55 \times 10^{-3}\right)$ and the response to wounding $\left(\mathrm{P}=3.86 \times 10^{-3}\right)$. Functions in cluster 4 were implicated in the immune response, such as the humoral immune response $\left(\mathrm{P}=1.89 \times 10^{-3}\right)$ and the humoral immune response mediated by circulating immunoglobulin $\left(\mathrm{P}=3.12 \times 10^{-2}\right)$ (Table I).

The enriched KEGG pathways for upregulated genes are also listed in Table I, including the cell cycle $\left(\mathrm{P}=3.32 \times 10^{-11}\right)$, extracellular matrix-receptor interaction $\left(\mathrm{P}=1.75 \times 10^{-7}\right)$ and DNA replication $\left(\mathrm{P}=2.74 \times 10^{-6}\right)$. Additionally, pathway enrichment analysis was conducted for downregulated genes. As shown in Table I, pathways such as those for the metabolism of xenobiotics by cytochrome $\mathrm{P} 450\left(\mathrm{P}=1.82 \times 10^{-4}\right)$, drug metabolism $\left(\mathrm{P}=2.25 \times 10^{-4}\right)$ and retinol metabolism $\left(\mathrm{P}=4.45 \times 10^{-3}\right)$ were enriched (Table I).

PPI network and module analysis. The PPI network for upregulated genes had 279 nodes and 5,690 interactions. In particular, upregulated mitotic arrest deficient 2-like 1 (MAD2L1; degree $=133)$, replication factor $\mathrm{C} 4$ (degree $=130)$, proliferating cell nuclear antigen $(P C N A$; degree $=125)$, cyclin-dependent kinase $1($ degree $=124)$ and cyclin B1 $(C C N B 1$; degree $=115)$ exhibited higher degrees in the PPI network. Modules 1 (Fig. 1) and 2 (Fig. 2) were obtained 
Table I. Enriched GO functions and KEGG pathways for the upregulated and downregulated genes ${ }^{\mathrm{a}}$.

A, Enriched GO functions for the upregulated genes

\begin{tabular}{|c|c|c|c|c|c|}
\hline Category & Term & Description & Gene no. & Gene symbol $^{\mathrm{b}}$ & P-value \\
\hline BP & GO:0000279 & M phase & 53 & DBF4, KNTC1, TTK & $5.27 \times 10^{-30}$ \\
\hline BP & GO:0022402 & Cell cycle process & 66 & DBF4, KNTC1, TTK & $3.34 \times 10^{-29}$ \\
\hline BP & GO:0007017 & Microtubule-based process & 28 & KIF23, CAV1, KIF4A & $1.09 \times 10^{-11}$ \\
\hline BP & GO:0000226 & Microtubule cytoskeleton organization & 21 & KIF23, CAV1, KIF11 & $7.27 \times 10^{-11}$ \\
\hline $\mathrm{BP}$ & GO:0007346 & Regulation of mitotic cell cycle & 19 & CDC7, DLGAP5, TIPIN & $6.43 \times 10^{-9}$ \\
\hline $\mathrm{BP}$ & GO:0010564 & Regulation of cell cycle process & 13 & CDC7, DLGAP5, TIPIN & $7.79 \times 10^{-6}$ \\
\hline $\mathrm{BP}$ & GO:0006259 & DNA metabolic process & 41 & UNG, DBF4, TIPIN & $1.80 \times 10^{-12}$ \\
\hline BP & GO:0006974 & Response to DNA damage stimulus & 28 & UNG, TIPIN, PRKDC & $5.83 \times 10^{-8}$ \\
\hline
\end{tabular}

B, Enriched GO functions for the downregulated genes

\begin{tabular}{|c|c|c|c|c|c|}
\hline Category & Term & Description & Gene no. & Gene symbol $^{\mathrm{b}}$ & P-value \\
\hline $\mathrm{BP}$ & GO:0030855 & Epithelial cell differentiation & 16 & ELF3, FOXA1, ANXA1 & $4.04 \times 10^{-7}$ \\
\hline $\mathrm{BP}$ & GO:0030216 & Keratinocyte differentiation & 7 & SPRRIA, PPL, CNFN & $3.48 \times 10^{-3}$ \\
\hline $\mathrm{BP}$ & GO:0010817 & Regulation of hormone levels & 12 & FAM3B, FOXA1, DUOX2 & $5.75 \times 10^{-4}$ \\
\hline $\mathrm{BP}$ & GO:0034754 & Cellular hormone metabolic process & 5 & DHRS9, UGT1A6, UGT1A10 & $4.25 \times 10^{-2}$ \\
\hline $\mathrm{BP}$ & GO:0002526 & Acute inflammatory response & 9 & $F 3, C L U, S A A 4$ & $1.55 \times 10^{-3}$ \\
\hline $\mathrm{BP}$ & GO:0009611 & Response to wounding & 23 & S100A9, ANXA1, PRDX5 & $3.86 \times 10^{-3}$ \\
\hline $\mathrm{BP}$ & GO:0006959 & Humoral immune response & 8 & $C F B, F O X J 1, C L U$ & $1.89 \times 10^{-3}$ \\
\hline $\mathrm{BP}$ & GO:0002455 & $\begin{array}{l}\text { Humoral immune response mediated } \\
\text { by circulating immunoglobulin }\end{array}$ & 4 & $C 7, C D 55, C R 2$ & $3.12 \times 10^{-2}$ \\
\hline
\end{tabular}

C, Enriched KEGG pathways for the upregulated and downregulated genes

\begin{tabular}{|c|c|c|c|c|c|c|}
\hline Regulation & Category & Term & Description & Gene no. & Gene symbol $^{\mathrm{b}}$ & P-value \\
\hline \multirow[t]{5}{*}{ Up } & KEGG & 04110 & Cell cycle & 21 & COL4A2, COL4A1, COL3A1 & $3.32 \times 10^{-11}$ \\
\hline & KEGG & 04512 & ECM-receptor interaction & 14 & COL4A2, COL4A1, COL3A1 & $1.75 \times 10^{-7}$ \\
\hline & KEGG & 03030 & DNA replication & 9 & RFC5, DNA2, RFC3 & $2.74 \times 10^{-6}$ \\
\hline & KEGG & 03430 & Mismatch repair & 7 & RFC5, EXO1, MSH6 & $1.92 \times 10^{-5}$ \\
\hline & KEGG & 04115 & p53 signaling pathway & 9 & CCNE2, CCNB1, CDK1 & $3.32 \times 10^{-4}$ \\
\hline \multirow[t]{5}{*}{ Down } & KEGG & 00980 & $\begin{array}{l}\text { Metabolism of xenobiotics } \\
\text { by cytochrome } \mathrm{P} 450\end{array}$ & 8 & GSTA1, GSTA3, ADH1C & $1.82 \times 10^{-4}$ \\
\hline & KEGG & 00982 & Drug metabolism & 8 & GSTA3, ADH1C, ADH7 & $2.25 \times 10^{-4}$ \\
\hline & KEGG & 00830 & Retinol metabolism & 6 & UGT1A1, UGT1A7, ALDH1A1 & $4.45 \times 10^{-3}$ \\
\hline & KEGG & 00590 & Arachidonic acid metabolism & 5 & AKR1C3, GGT6, ALOX15 & $2.61 \times 10^{-2}$ \\
\hline & KEGG & 04640 & Hematopoietic cell lineage & 6 & $M S 4 A 1, C D 1 C, C D 1 D$ & $2.96 \times 10^{-2}$ \\
\hline
\end{tabular}

${ }^{a}$ The enriched GO terms listed in the table are the functions for the top four clusters with the highest enriched score. ${ }^{\mathrm{b} O n l y}$ the most relevant genes in each category are listed.GO, Gene Ontology; KEGG, Kyoto Encyclopedia of Genes and Genomes; ECM, extracellular matrix; BP, biological process. KNTC, kinetochore associated; KIF, kinesin family; CAV, caveolin; CDK, cyclin-dependent kinase; CDC, cell division cycle; DLGAP, discs large homolog associated protein; TIPIN timeless-interacting protein; UNG, uracil DNA glycosylase; PRKDC, protein kinase, DNA-activated, catalytic polypeptide; SPRR, small proline rich protein; PPL, periplakin; CNFN, cornifelin; DHRS, dehydrogenase/ reductase (SDR family); UGT, UDP glucuronosyltransferase; ELF3, E74-like ETS transcription factor 3; FOX, forkhead box; ANX, annexin; FAM, family with sequence similarity; DUOX, dual oxidase; F3, coagulation factor III, tissue factor; CLU, clusterin; SAA4, serum amyloid A4, constitutive; S100A9, S100 calcium binding protein A9; PRDX, peroxiredoxin; CFB, complement factor B; C7, complement component 7; CD, cluster of differentiation; CR2, complement component 3d receptor 2; COL, collagen; RFC, replication factor C; DNA2, DNA replication helicase/nuclease 2; EXO, exonuclease; MSH, mutS homolog; CCN, cyclin; GSTA, glutathione S-transferase alpha; ADH, alcohol dehydrogenase; AKR, aldo-keto reductase; GGT, gamma-glutamyltransferase; ALOX15, arachidonate 15-lipoxygenase; MS4A1, membrane spanning 4-domains A1. 


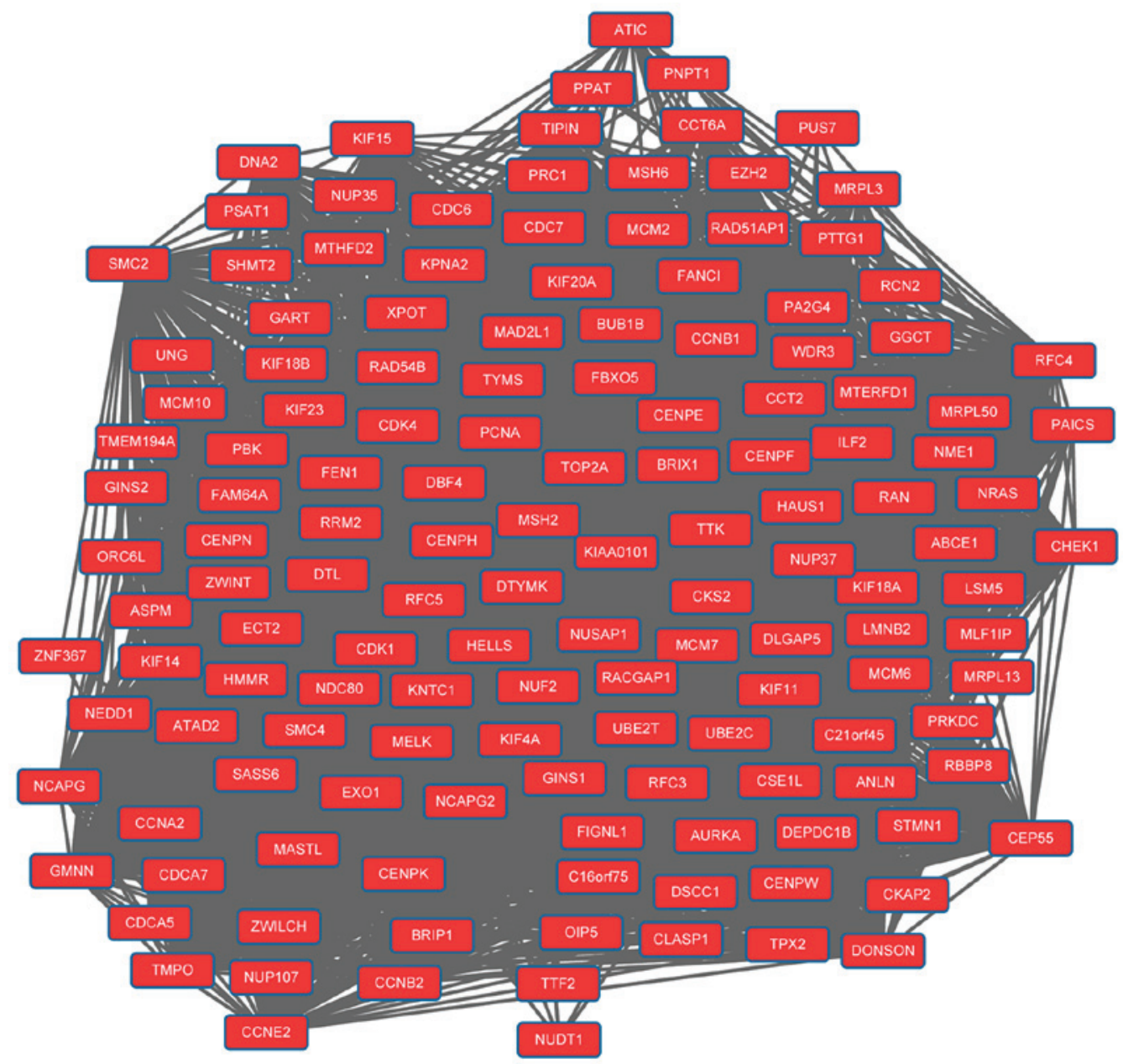

Figure 1. Module 1 obtained from the protein-protein interaction network of upregulated genes.

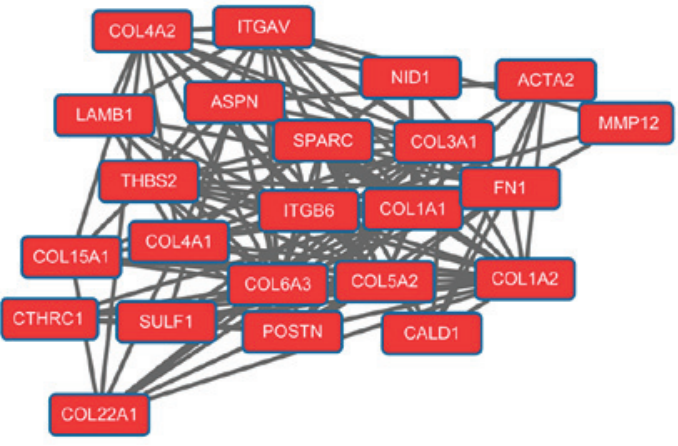

Figure 2. Module 2 obtained from the protein-protein interaction network of upregulated genes.

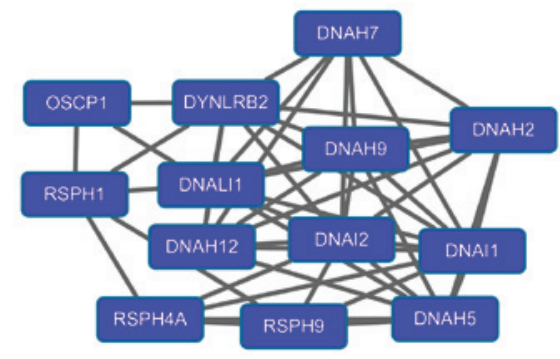

Figure 3. Module 3 obtained from the protein-protein interaction network of downregulated genes.

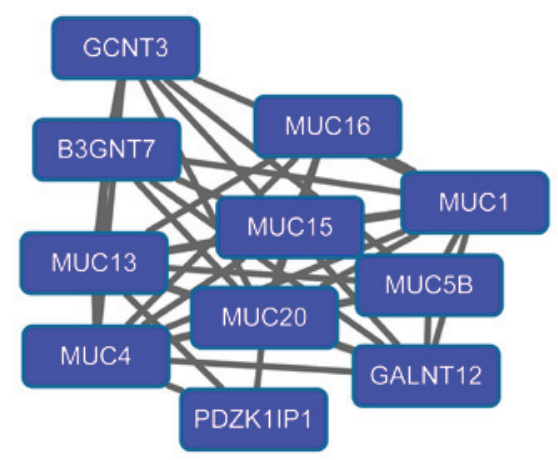

Figure 4. Module 4 obtained from the protein-protein interaction network of downregulated genes.

from the PPI network of upregulated genes. Module 1 had 144 nodes and 5,091 interactions. Module 2 had 23 nodes and 133 interactions. The cell cycle was an enriched pathway for the DEGs in modules 1 and 2.

The PPI network for downregulated genes had 260 nodes and 517 interactions. Importantly, downregulated glutathione S-transferase $\alpha 1$ (GSTA1; degree=19), GSTA3 (degree=19), dynein, light chain, roadblock-type 2 (degree=17), calmodulin 1 (degree=16), member A1 of aldehyde dehydrogenase $1(A L D H 1 A 1$; degree $=15)$ and sperm-associated 
antigen $6($ degree $=15)$ had higher degrees in the PPI network. Modules 3 (Fig. 3) and 4 (Fig. 4) were obtained from the PPI network of downregulated genes. Module 3 had 13 nodes and 48 interactions. The enriched pathway for DEGs in module 3 included the mitogen-activated protein kinase signaling pathway. Module 4 had 11 nodes, such as mucin 1 (MUCl), and 43 interactions. O-glycan biosynthesis was an enriched pathway for DEGs in module 4.

\section{Discussion}

In the present study, 951 DEGs were screened in the NPC samples compared with normal healthy nasopharyngeal tissue samples. Function enrichment indicated that the upregulated genes were mainly associated with the cell cycle, cytoskeleton organization and DNA metabolism. The downregulated genes were associated with cell differentiation, hormone metabolism, the inflammatory response and the immune response. Meanwhile, upregulated MAD2L1 (degree=133), PCNA (degree=125) and $C C N B 1$ (degree=115), and downregulated ALDH1Al (degree=15) exhibited higher degrees of interaction in the PPI network.

Via the induction of mitotic arrest, MAD2 can cause chemosensitization to cisplatin in NPC cells and activate the apoptosis pathway (24). The aberrantly reduced expression of $M A D 2$ can lead to a defective mitotic checkpoint and promote chromosomal instability in the disease (25). Sensitization to vincristine induced by $M A D 2$ is associated with $\mathrm{Raf} / \mathrm{Bcl}-2$ phosphorylation and mitotic arrest in NPC cells (26). The M-phase events of $M A D 2$ and $C C N B 1 / C D C 2$ activation are essential to the paclitaxel-induced apoptosis of human NPC cells (27). Thus, the expression levels of MAD2L1 and $C C N B 1$ may be associated with NPC. It has been reported that upregulated $B C L-2$ and a high $P C N A$ labeling index may be implicated in local recurrence in NPC patients receiving the primary treatment of radiation therapy (28). Through inhibiting the expression of PCNA, a PCNA-small interfering RNA compound can effectively interfere with NPC cells and may be used in the gene therapy of NPC (29). These data indicate that PCNA may also play a role in NPC.

The expression of $A L D H 1$ in the invasive front links, which is associated with tumor aggressiveness and epithelial-mesenchymal transition characteristics, may serve as a promising predictive factor in NPC $(30,31)$. Budding cells characterized by a high level of $A L D H 1$ may have invasive and metastatic properties, therefore, the degree of expression can be a useful prognostic marker in NPC patients (32). It has been reported that the overexpression of ALDH1A1 in NPC is associated with enhanced invasiveness (33). These data indicate that ALDHIAl may have a close correlation with NPC. $M U C 1$, a mucinous glycoprotein required for the detachment and release of tumor cells, combined with other invasiveness and angiogenic factors may function in a complex sequential process that ends in the metastasis of EBV-infected NPC cells (34). This may indicate that the expression level of $M U C 1$ is associated with EBV-associated NPC.

In conclusion, in the present study, an integrated bioinformatics analysis of genes that may be involved in EBV-associated NPC was performed. A total of 951 DEGs were screened in the NPC samples compared with the normal healthy nasopharyngeal tissue samples. Certain DEGs, such as MAD2L1, CCNB1, PCNA, ALDHIAl and MUC1, may have a correlation with NPC. However, future studies are required to advance the understanding of their mechanisms of action in NPC.

\section{Acknowledgements}

This study was supported by the Fundamental Research Funds for the Central Universities (grant no. 20620140692), the Jiangsu Government Scholarship for Overseas Studies 2014, the Medical Youth Priming Project of Nanjing (grant no. QYK11162), the National Natural Science Foundation of China (grant no. 30973302), the Medical Important People Project of Jiangsu Province (grant no. RC2007010) and the Medical Important Developing Project of Nanjing (grant no. ZKX06019).

\section{References}

1. Xia H, Ng SS, Jiang S, Cheung WK, Sze J, Bian XW, Kung HF and Lin MC: miR-200a-mediated downregulation of ZEB2 and CTNNB1 differentially inhibits nasopharyngeal carcinoma cell growth, migration and invasion. Biochem Biophys Res Commun 391: 535-541, 2010.

2. Zhang $\mathrm{F}$ and Zhang J: Clinical hereditary characteristics in nasopharyngeal carcinoma through Ye-Liang's family cluster. Chin Med J (Engl) 112: 185-187, 1999.

3. Lo KW, Chung GT and To KF: Deciphering the molecular genetic basis of NPC through molecular, cytogenetic, and epigenetic approaches. Semin Cancer Biol 22: 79-86, 2012.

4. Yu MC, Ho JH, Lai SH and Henderson BE: Cantonese-style salted fish as a cause of nasopharyngeal carcinoma: Report of a case-control study in Hong Kong. Cancer Res 46: 956-961, 1986.

5. Lozano R, Naghavi M, Foreman K, Lim S, Shibuya K, Aboyans V, Abraham J, Adair T, Aggarwal R, Ahn SY, et al: Global and regional mortality from 235 causes of death for 20 age groups in 1990 and 2010: A systematic analysis for the global burden of disease study 2010. Lancet 380: 2095-2128, 2012.

6. Wei MX, Moulin JC, Decaussin G, Berger F and Ooka T: Expression and tumorigenicity of the Epstein-Barr virus BARF1 gene in human Louckes B-lymphocyte cell line. Cancer Res 54: 1843-1848, 1994.

7. Decaussin G, Sbih-Lammali F, de Turenne-Tessier M, Bouguermouh A and Ooka T: Expression of BARF1 gene encoded by Epstein-Barr virus in nasopharyngeal carcinoma biopsies. Cancer Res 60: 5584-5588, 2000.

8. Seto E, Yang L, Middeldorp J, Sheen TS, Chen JY, Fukayama M, Eizuru Y, Ooka T and Takada K: Epstein-Barr virus (EBV)-encoded BARF1 gene is expressed in nasopharyngeal carcinoma and EBV-associated gastric carcinoma tissues in the absence of lytic gene expression. J Med Virol 76: 82-88, 2005.

9. Zhou L, Jiang W, Ren C, Yin Z, Feng X, Liu W, Tao Q and Yao K: Frequent hypermethylation of RASSF1A and TSLC1, and high viral load of Epstein-Barr Virus DNA in nasopharyngeal carcinoma and matched tumor-adjacent tissues. Neoplasia 7: 809-815, 2005.

10. Lo KW, Kwong J, Hui AB, Chan SY, To KF, Chan AS, Chow LS, Teo PM, Johnson PJ and Huang DP: High frequency of promoter hypermethylation of RASSF1A in nasopharyngeal carcinoma. Cancer Res 61: 3877-3881, 2001.

11. Feng P, Ren EC, Liu D, Chan $\mathrm{SH}$ and $\mathrm{Hu} \mathrm{H}$ : Expression of Epstein-Barr virus lytic gene BRLF1 in nasopharyngeal carcinoma: Potential use in diagnosis. J Gen Virol 81: 2417-2423, 2000.

12. Horikawa T, Sheen TS, Takeshita H, Sato H, Furukawa M and Yoshizaki T: Induction of c-Met proto-oncogene by Epstein-Barr virus latent membrane protein-1 and the correlation with cervical lymph node metastasis of nasopharyngeal carcinoma. Am J Pathol 159: 27-33, 2001.

13. Heussinger N, Büttner M, Ott G, Brachtel E, Pilch BZ, Kremmer E and Niedobitek G: Expression of the Epstein-Barr virus (EBV)-encoded latent membrane protein 2A (LMP2A) in EBV-associated nasopharyngeal carcinoma. J Pathol 203: 696-699, 2004 
14. Sengupta S, den Boon JA, Chen IH, Newton MA, Dahl DB, Chen M, Cheng YJ, Westra WH, Chen CJ, Hildesheim A, et al: Genome-wide expression profiling reveals EBV-associated inhibition of MHC class I expression in nasopharyngeal carcinoma. Cancer Res 66: 7999-8006, 2006.

15. Gautier L, Cope L, Bolstad BM and Irizarry RA: Affy-analysis of Affymetrix GeneChip data at the probe level. Bioinformatics 20: 307-315, 2004.

16. Bolstad BM, Irizarry RA, Åstrand M and Speed TP: A comparison of normalization methods for high density oligonucleotide array data based on variance and bias. Bioinformatics 19: 185-193, 2003.

17. Smyth GK: Limma: Linear models for microarray data. In Bioinformatics and Computational Biology Solutions using $\mathrm{R}$ and Bioconductor. Gentleman R, Carey V, Dudoit S, Irizarry R and Huber W (eds), Springer, New York, pp397-420, 2005.

18. Tweedie S, Ashburner M, Falls K, Leyland P, McQuilton P, Marygold S, Millburn G, Osumi-Sutherland D, Schroeder A, Seal R, et al: FlyBase: Enhancing Drosophila gene ontology annotations. Nucleic Acids Res 37: D555-D559, 2009.

19. Kanehisa M and Goto S: KEGG: kyoto encyclopedia of genes and genomes. Nucleic Acids Res 28: 27-30, 2000.

20. Dennis G Jr, Sherman BT, Hosack DA, Yang J, Gao W, Lane HC and Lempicki RA: DAVID: Database for annotation, visualization, and integrated discovery. Genome Biol 4: P3, 2003.

21. Franceschini A, Szklarczyk D, Frankild S, Kuhn M, Simonovic M Roth A, Lin J, Minguez P, Bork P, von Mering C and Jensen LJ: STRING v9. 1: Protein-protein interaction networks, with increased coverage and integration. Nucleic Acids Res 41: D808-D815, 2013

22. Smoot ME, Ono K, Ruscheinski J, Wang PL and Ideker T: Cytoscape 2.8: New features for data integration and network visualization. Bioinformatics 27: 431-432, 2011.

23. Nepusz T, Yu H and Paccanaro A: Detecting overlapping protein complexes in protein-protein interaction networks. Nat Methods 9: 471-472, 2012.

24. Cheung HW, Jin DY, Ling MT, Wong YC, Wang Q, Tsao SW and Wang X: Mitotic arrest deficient 2 expression induces chemosensitization to a DNA-damaging agent, cisplatin, in nasopharyngeal carcinoma cells. Cancer Res 65: 1450-1458, 2005
25. Wang X, Jin DY, Wong YC, Cheung AL, Chun AC, Lo AK, Liu Y and Tsao SW: Correlation of defective mitotic checkpoint with aberrantly reduced expression of MAD2 protein in nasopharyngeal carcinoma cells. Carcinogenesis 21: 2293-2297, 2000.

26. Wang $X$, Jin DY, Wong HL, Feng H, Wong YC and Tsao SW: MAD2-induced sensitization to vincristine is associated with mitotic arrest and Raf/Bcl-2 phosphorylation in nasopharyngeal carcinoma cells. Oncogene 22: 109-116, 2003.

27. Huang TS, Shu CH, Chao Y, Chen SN and Chen LL: Activation of MAD 2 checkprotein and persistence of cyclin B1/CDC 2 activity associate with paclitaxel-induced apoptosis in human nasopharyngeal carcinoma cells. Apoptosis 5: 235-241, 2000.

28. Tsai ST, Jin YT, Leung HW, Wang ST, Tsao CJ and Su IJ: Bcl-2 and proliferating cell nuclear antigen (PCNA) expression correlates with subsequent local recurrence in nasopharyngeal carcinomas. Anticancer Res 18: 2849-2854, 1998.

29. Lian B, Wang JQ and Jin L: Effects of siRNA targeting PCNA gene on nasopharyngeal carcinoma CNE2 cell cycle. Chin J Pathophysiol 25: 1533-1537, 2009 (In Chinese).

30. Luo WR and Yao KT: Cancer stem cell characteristics, ALDH1 expression in the invasive front of nasopharyngeal carcinoma. Virchows Arch 464: 35-43, 2014.

31. Wu A, Luo W, Zhang Q, Yang Z, Zhang G, Li S and Yao K: Aldehyde dehydrogenase 1 , a functional marker for identifying cancer stem cells in human nasopharyngeal carcinoma. Cancer Lett 330: 181-189, 2013.

32. Luo WR, Gao F, Li SY and Yao KT: Tumour budding and the expression of cancer stem cell marker aldehyde dehydrogenase 1 in nasopharyngeal carcinoma. Histopathology 61: 1072-1081, 2012.

33. Hou W, He W, Li Y, Ma R, Wang Z, Zhu X, Fu Q, Wen Y, Li H and Wen W: Increased expression of aldehyde dehydrogenase 1 A1 in nasopharyngeal carcinoma is associated with enhanced invasiveness. Eur Arch Otorhinolaryngol 271: 171-179, 2014.

34. Kondo S, Yoshizaki T, Wakisaka N, Horikawa T, Murono S, Jang KL, Joab I, Furukawa M and Pagano JS: MUC1 induced by Epstein-Barr virus latent membrane protein 1 causes dissociation of the cell-matrix interaction and cellular invasiveness via STAT signaling. J Virol 81: 1554-1562, 2007. 